\title{
Competencias pedagógicas desde la teoría kaizen
}

\author{
Sonia Carmina Venegas Paz \\ punto_maximo_77@ hotmail.com \\ Universidad Cesar Vallejo, Piura-Perú \\ Patricia Mercedes Tapia Macías \\ Patty_tapia56@yahoo.com \\ Universidad Cesar Vallejo, Piura-Perú \\ Marybel Mantilla Crespo \\ marybelmantilla@gmail.com \\ Rowan Collage at Burlington County/ \\ in Mount Laurel Township, $\mathrm{Nj}$. \\ María Pía Da Silva \\ piadasilva89@hotmail.com \\ Universitat de les Illes Balears
}

\section{RESUMEN}

La teoría Kaizen en educación, tiene un impacto positivo en el desarrollo de las competencias pedagógicas en los docentes, despierta el interés de los estudiantes en cada lección, promoviendo el pensamiento crítico y mejorando el conocimiento de conceptos específicos. La investigación plantea como objetivo general determinar que una propuesta basada en la teoría Kaizen, fortalece las competencias pedagógicas en los docentes de Historia y Geografía en Guayaquil - 2021. La metodología fue de tipo experimental con diseño pre-experimental. La población estuvo constituida por 100 docentes del área de Historia y Geografía de distintos centros educativos; se realizó un muestreo no probabilístico (intencional), donde la muestra quedó conformada por 50 docentes. Para la técnica de recolección de datos, se utilizó la encuesta y como instrumento el cuestionario; se elaboró un análisis descriptivo e inferencial de los resultados. Se obtuvo como conclusiones que la propuesta basada en la teoría Kaizen influye de manera positiva en el fortalecimiento de competencias pedagógicas, donde los valores del postest fueron superiores al pretest; asimismo, la propuesta basada en teoría Kaizen fortalece las competencias didácticas, productivas, interactivas, investigativas y para la dirección en los docentes.

Palabras clave: competencias pedagógicas; teoría kaizen; investigación. 


\title{
Pedagogical competences from the kaizen theory
}

\begin{abstract}
Kaizen theory in education has a positive impact on the result, as it awakens the interest of students in each lesson, promotes critical thinking and improves knowledge of specific concepts. The present research work raises as a general objective to determine that a proposal based on Kaizen theory strengthens the pedagogical competences in History and Geography teachers in Guayaquil - 2021. The methodology was experimental with a preexperimental design. The population consisted of 100 teachers from the area of History and Geography from different educational centers; A non-probabilistic (intentional) sampling was carried out, where the sample was made up of 50 teachers. Regarding the data collection technique, the survey was used and the questionnaire was used as an instrument; A descriptive and inferential analysis of the results was carried out. The conclusions were obtained: the proposal based on Kaizen theory positively influences the strengthening of pedagogical competences, where the post-test values were higher than the pre-test; Likewise, the proposal based on Kaizen theory strengthens the didactic, productive, interactive, investigative and management competencies in teachers.
\end{abstract}

Keywords: pedagogical competences; kaizen theory; research.

Artículo recibido: 30 noviembre. 2021 Aceptado para publicación: 29 diciembre 2021 Correspondencia:punto_maximo_77@hotmail.com Conflictos de Interés: Ninguna que declarar 


\section{INTRODUCCIÓN}

Fortalecer las competencias pedagógicas docentes se ha vuelto un punto de gran trascendencia en lo que concierne a la calidad educativa (Escobar, 2005). Según (Gutiérrez, 2003) día a día incrementa la búsqueda del mejoramiento por las instituciones escolares; este desafío corresponde al pedido de una sociedad cada vez más exigente. Desde la perspectiva internacional la UNESCO ha considerado que los educadores son pieza clave en la búsqueda de la equidad y calidad en el ámbito educativo, no obstante, su formación es motivo aún de preocupación. De igual forma, su Instituto de Estadística (IUE), hace referencia a que existe escasez de maestros plenamente preparados a nivel mundial. Debido a esto, para el año 2030 deberían estar capacitados unos 69 millones de docentes con el propósito de asegurar la universalización de la enseñanza (UNESCO, 2021). A nivel latinoamericano y caribeño, según el estudio realizado por Bruns y Luque (2014), se comprobó que existe muy poca correspondencia entre las capacidades que un docente debería tener y las que posee realmente; así, en países como Colombia, Ecuador o Chile solo un $3 \%$ de estos profesionales superaron las pruebas que validan cuanto saben los maestros.

Respecto al ámbito nacional, en Ecuador, el informe del Instituto Nacional de Evaluación Educativa INEVAL (2018), expresó que el proceso concerniente a renovación y especialización del talento docente, debe aumentar su rango de acción, especialmente en zonas rurales. Para Faidutti (2018, citado por Rengifo y Espinoza, 2019), existe un gran número de educadores que no están debidamente preparados y son mal remunerados. Según la investigación de Suasnabas y Juárez (2020), entre los problemas que acrecientan una educación deficiente en el Ecuador se encuentran: debilidad en la preparación de los maestros, centralización y salarios bajos. En este orden, considerando que las competencias pedagógicas se constituyen en el pilar de la destreza de los docentes, se plantea la siguiente pregunta ¿De qué forma una propuesta basada en la teoría Kaizen, fortalece las competencias pedagógicas?

La investigación tiene justificación teórica, debido a que amplió la cantidad de información objetiva existente sobre las variables de estudio: teoría Kaizen y competencias pedagógicas docentes. Los resultados obtenidos pueden incorporarse como conocimiento a las ciencias de la educación siendo de valor significativo en la localidad de Guayaquil por existir escasos trabajos que relacionen ambas variables. El aporte 
metodológico del trabajo, permitió la elaboración de un instrumento válido y confiable, con la finalidad de identificar de qué forma la teoría Kaizen contribuye en el fortalecimiento de las competencias pedagógicas en los educadores de Historia y Geografía; dando como resultado una propuesta sustentable y sostenible.

La aplicación de la propuesta de mejoramiento continuo, se reflejó en un ejercicio profesional de calidad y desarrollo personal. En este sentido, el estudio sentó las bases para la creación de estrategias específicas que procuraron el desarrollo formativo del docente en el área de Historia y Geografía, generando soluciones a las dificultades que pudiesen surgir en la praxis educativa. Se destaca la motivación del maestro para buscar permanentemente la innovación y perfeccionamiento; sensibilizando la importancia del mejoramiento continuo y fortalecimiento de competencias pedagógicas. Un maestro no sólo brinda conocimiento, transmite valores, aspiraciones e inspiraciones. Este trabajo focalizó su atención en variables psico-sociales, relacionadas en gran manera al bienestar humano.

Por su lado, el Objetivo General se basó en: Determinar que una propuesta basada en la teoría Kaizen, fortalece las competencias pedagógicas en los docentes en Guayaquil 2021. Con relación a los objetivos específicos se planteó: 1) Establecer que una propuesta basada en la teoría Kaizen, fortalece la competencia didáctica; 2) Comprobar que una propuesta basada en la teoría Kaizen, fortalece la competencia productiva; 3) Demostrar que una propuesta basada en la teoría Kaizen, fortalece la competencia interactiva; 4) Verificar que una propuesta basada en la teoría Kaizen, fortalece la competencia investigativa; y, 5) Determinar que una propuesta basada en la teoría Kaizen fortalece la competencia para la dirección. Como Hipótesis General se plantea que existe relación significativa entre la propuesta basada en la teoría Kaizen, y el fortalecimiento de las competencias pedagógicas en los docentes de Historia y Geografía en Guayaquil - 2021.

\section{ESTRATEGIAS METODOLÓGICAS O MATERIALES Y MÉTODOS}

La presente investigación tuvo un enfoque cuantitativo, de tipo experimental-aplicada, puesto que su objetivo fue resolver un determinado problema, enfocándose en la búsqueda y consolidación del conocimiento para su aplicación (Arias, 2020). En cuanto al nivel, la investigación experimental es netamente explicativa; el diseño fue preexperimental, con un modelo de pre-prueba/post-prueba con un solo grupo. Este diseño se diagrama así: 


\section{GE: $\quad 01 \quad X \quad 02$}

La población fue de 100 educadores de Historia y Geografía de diferentes planteles educativos, distribuidos en 60 personas de sexo femenino y 40 personas de sexo masculino. El muestreo fue no probabilístico, basado en la elección de los miembros según el criterio específico de la investigadora. Para los criterios de inclusión, se consideraron los docentes de Historia y Geografía de sexo femenino y masculino, que laboran en el nivel de Educación Básica y Media en instituciones públicas. Para los criterios de exclusión, considerando la gran población de educadores de Historia y Geografía de la Provincia Guayas, se tomaron en cuenta sólo aquellos pertenecientes a colegios públicos de Guayaquil. La distribución de la muestra quedó conformada en 50 personas, 20 de sexo masculino y 30 de sexo femenino.

Hernández et. al., (2010), manifestaron que recolectar datos implica tres actividades relacionadas estrechamente: seleccionar un instrumento, aplicarlo y preparar las observaciones, registros y dimensiones obtenidas. Así, para la recolección de datos se utilizó la encuesta, siendo el instrumento un cuestionario de escala Likert, organizado en 4 puntos donde cada opción se denota con un número: (1) Nunca, (2) Pocas Veces, (3) Frecuentemente y (4) Siempre. Se validó a través del método de juicio de expertos, útil para verificar la fiabilidad de una investigación; siendo sometido a la experticia de cinco jueces, quienes revisaron todos los reactivos para garantizar que el mismo mida la variable de estudio, considerándolo aplicable por unanimidad. La validez de criterio se realizó con una prueba piloto en la Unidad Educativa Calicuchima, con la que se obtuvo la correlación de Pearson en base a los puntajes derivados del instrumento y los criterios específicos escogidos. Adicionalmente, para la validez de constructo, se procedió a un análisis factorial posterior a la aplicación de la prueba piloto. Seguidamente, para obtener la confiabilidad del instrumento, se utilizó el índice Alfa de Cronbach por medio del paquete estadístico SPSS, mismo que tuvo como resultado 0,794 que se clasifica como una confiabilidad aceptable.

En la aplicación del instrumento para recolectar información, se procedió a la realización del análisis de los resultados a través de la codificación y tabulación de los datos, organizándolos en cuadros de distribución de frecuencias y porcentajes, en base a los valores establecidos. De tal forma, como técnica de procesamiento se seleccionó la 
estadística descriptiva, que permitió un análisis metódico; asimismo, la estadística inferencial, con el propósito de obtener conclusiones útiles para hacer deducciones sobre una totalidad, se utilizó el programa Excel y el paquete estadístico SPSS.

En lo que se refiere a los aspectos éticos, se encuentran los siguientes: la protección de los participantes, para lo cual las encuestas fueron anónimas; se respetó la autonomía de los docentes, informándoles de los fines que se perseguían con el estudio, sin ningún tipo de coacción económica o de poder. Igualmente, se tomaron en cuenta las normas APA, el sistema Turnitin (no plagio) y las recomendaciones para la realización de investigación científica de la Universidad César Vallejo. Cabe mencionar, que se contó con la autorización previa del personal directivo de cada uno de los centros educativos donde se desarrolló la investigación.

\section{RESULTADOS Y DISCUSIÓN}

\section{Análisis descriptivo}

\section{Objetivo General}

- Determinar que una propuesta basada en la teoría Kaizen, fortalece las competencias pedagógicas en los docentes de Historia y Geografía en Guayaquil - 2021.

\section{Tabla 1}

Niveles de la variable competencias pedagógicas

Variable: Competencias pedagógicas

\begin{tabular}{lcccc}
\hline \multirow{2}{*}{ NIVEL } & & PRE TEST & \multicolumn{2}{c}{ POS TEST } \\
& F & \% & F & $\%$ \\
\hline Alto & 7 & 14 & 50 & 100 \\
Medio & 43 & 86 & 0 & 0 \\
Bajo & 0 & 0 & 0 & 0 \\
Total & 50 & 100 & 50 & 100 \\
\hline
\end{tabular}

Fuente: Cuestionario aplicado

\section{Figura 1}

Niveles de la variable competencias pedagógicas 


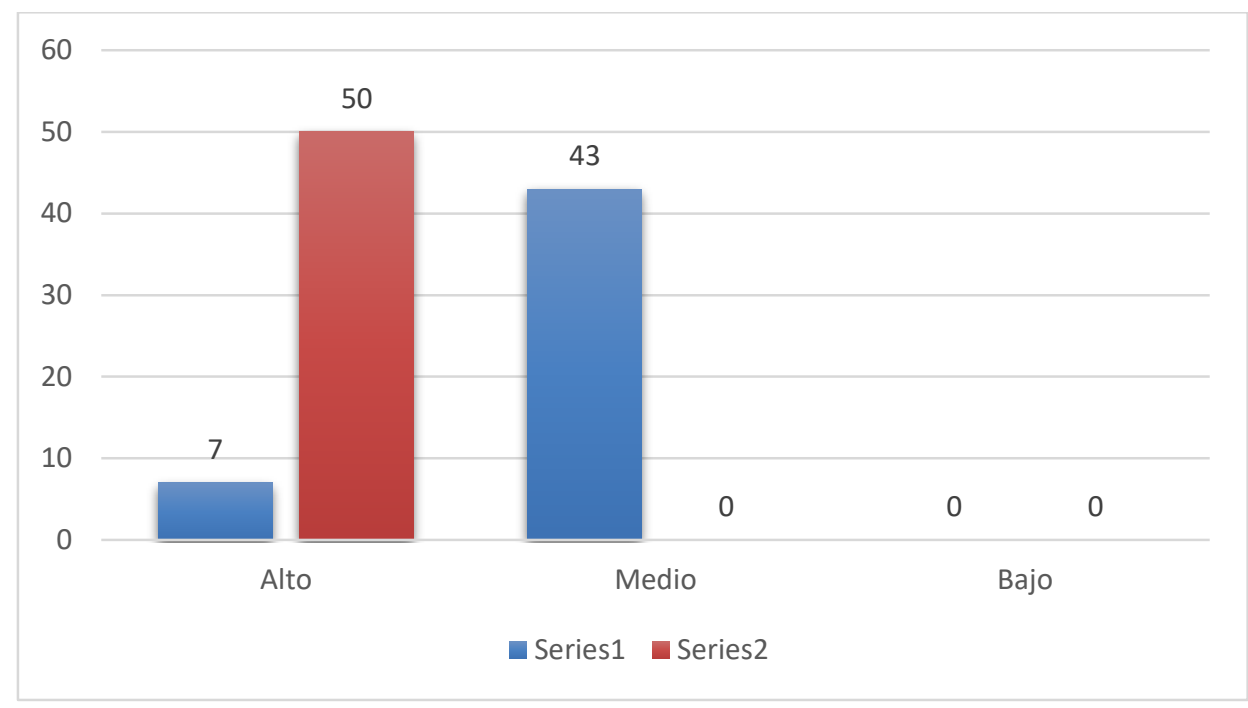

Fuente: Tabla 1

Interpretación: En la tabla 1 y figura 1, se aprecia que el $86 \%$ se encuentra en nivel medio, y el 14\% en el nivel alto en el pretest; luego se observa que el 100\% se ubica en nivel alto en el post test. Entonces se determina que la propuesta basada en la teoría Kaizen fortalece las competencias pedagógicas en docentes de Historia y Geografía de Guayaquil.

\section{Objetivo específico 1}

- Determinar que una propuesta basada en la teoría Kaizen, fortalece la competencia didáctica en los docentes de Historia y Geografía en Guayaquil - 2021.

\section{Tabla 2}

Niveles de la dimensión competencia didáctica

\begin{tabular}{lcccc}
\hline \multicolumn{1}{c}{ NIVEL } & \multicolumn{2}{c}{ PRE TEST } & \multicolumn{2}{c}{ POS TEST } \\
& F & \% & F & $\%$ \\
\hline Alto & 22 & 44 & 50 & 100 \\
Medio & 26 & 52 & 0 & 0 \\
Bajo & 2 & 4 & 0 & 0 \\
Total & 50 & 100 & 50 & 100 \\
\hline
\end{tabular}

Fuente: Cuestionario aplicado

\section{Figura 2}

Niveles de la dimensión competencia didáctica 


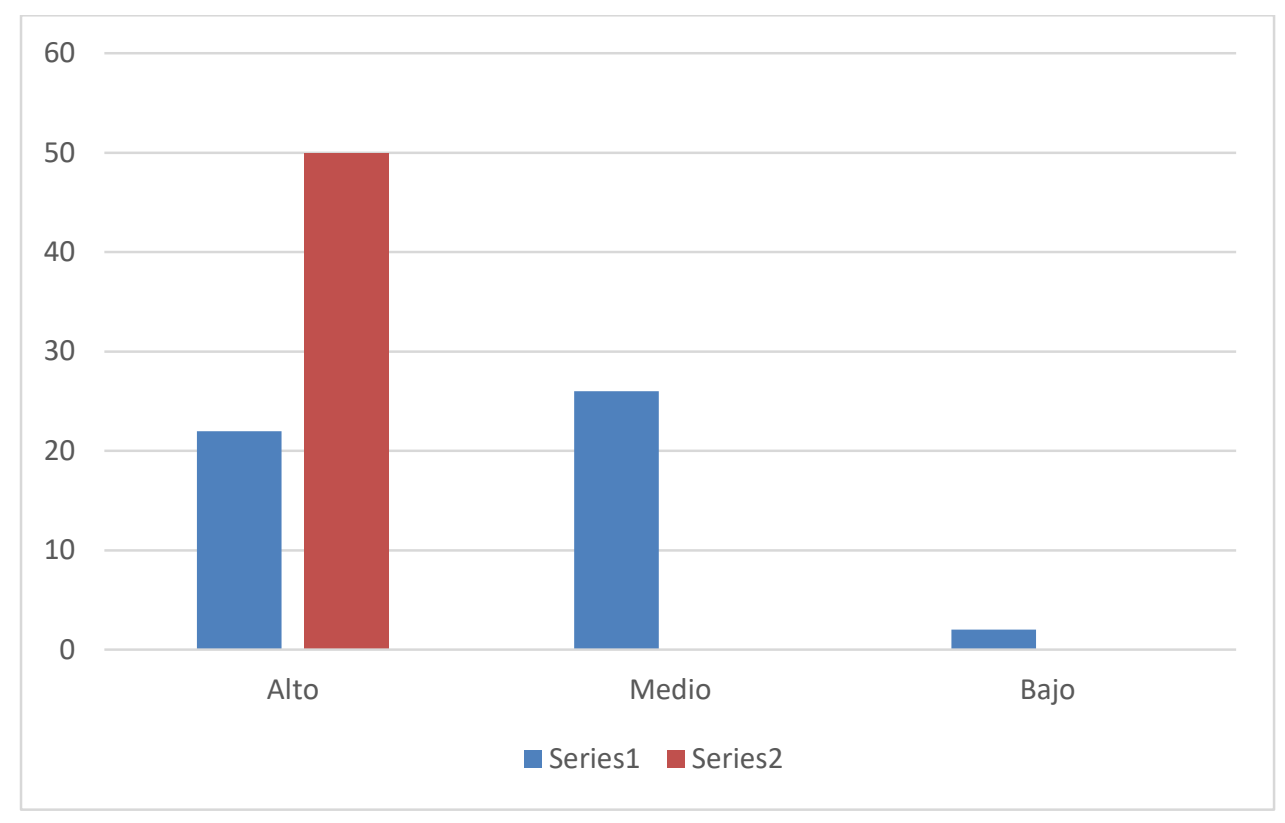

Fuente: Tabla 2

Interpretación: En la tabla 2 y figura 2, se distingue que el 52\% se encuentra en nivel medio, y el $44 \%$ en el nivel alto en el pre test; posteriormente se visualiza que el $100 \%$ se ubica en nivel alto en el post test. Por tanto, se determina que la propuesta basada en la teoría Kaizen fortalece la competencia didáctica en docentes de Historia y Geografía de Guayaquil.

\section{Objetivo específico 2}

- Comprobar que una propuesta basada en la teoría Kaizen, fortalece la competencia productiva en los docentes de Historia y Geografía en Guayaquil - 2021.

\section{Tabla 3}

Niveles de la dimensión competencia productiva

\begin{tabular}{ccccc}
\hline \multirow{2}{*}{ NIVEL } & \multicolumn{2}{c}{ PRE TEST } & \multicolumn{2}{c}{ POS TEST } \\
& F & $\%$ & F & $\%$ \\
\hline Alto & 9 & 18 & 50 & 100 \\
Medio & 40 & 80 & 0 & 0 \\
Bajo & 1 & 2 & 0 & 0 \\
Total & 50 & 100 & 50 & 100 \\
\hline
\end{tabular}

Fuente: Cuestionario aplicado

\section{Figura 3}

Niveles de la dimensión competencia productiva 


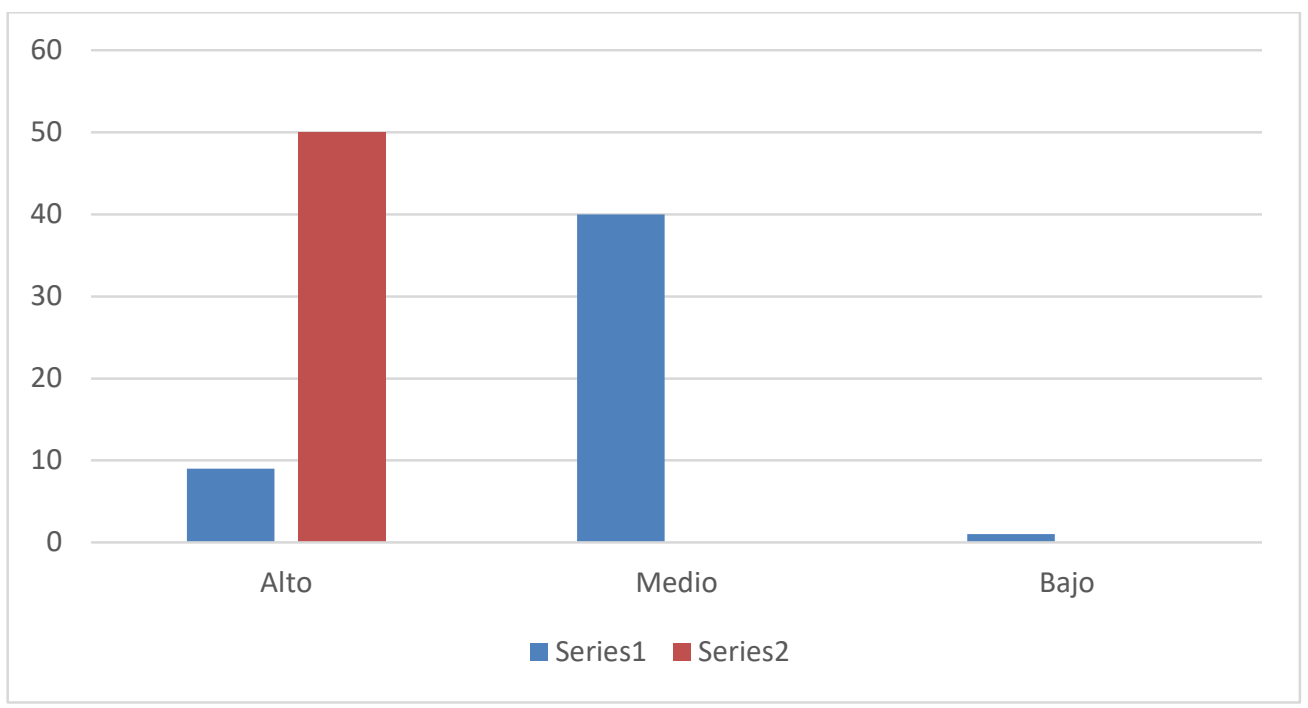

Fuente: Tabla 3

Interpretación: En la tabla 3 y figura 3, se observa que el $80 \%$ se encuentra en nivel medio, y el $18 \%$ en el nivel alto en la pre-prueba; luego se denota que el 100\% se ubica en nivel alto en el post test. De este modo, se determina que la propuesta basada en la teoría Kaizen fortalece la competencia productiva en docentes de Historia y Geografía de Guayaquil.

\section{Objetivo específico 3}

- Demostrar que una propuesta basada en la teoría Kaizen, fortalece la competencia interactiva en los docentes de Historia y Geografía en Guayaquil - 2021.

\section{Tabla 4}

Niveles de la dimensión competencia interactiva

\begin{tabular}{ccccc}
\hline \multirow{2}{*}{ NIVEL } & & PRE TEST & \multicolumn{2}{c}{ POS TEST } \\
& F & \% & F & $\%$ \\
\hline Alto & 6 & 12 & 50 & 100 \\
Medio & 42 & 84 & 0 & 0 \\
Bajo & 2 & 4 & 0 & 0 \\
Total & 50 & 100 & 50 & 100 \\
\hline
\end{tabular}

Fuente: Cuestionario aplicado

\section{Figura 4}

Niveles de la dimensión competencia interactiva 


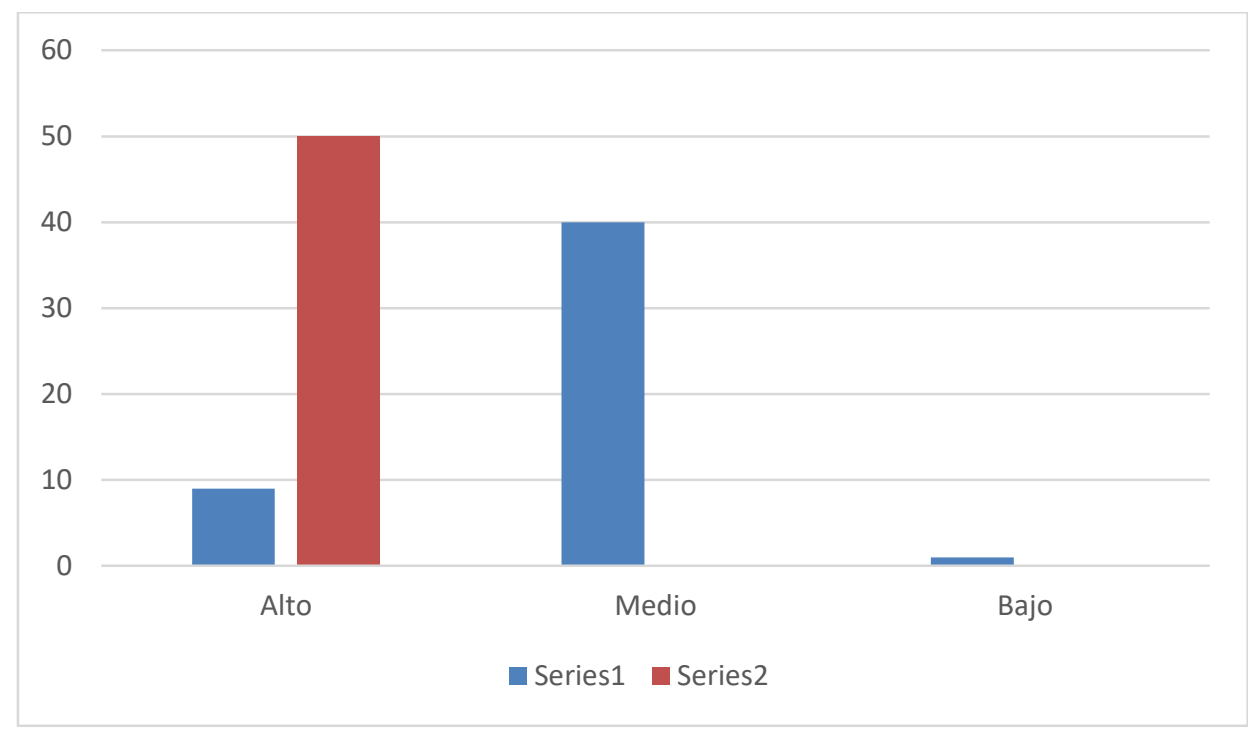

Fuente: Tabla 4

Interpretación: En la tabla 4 y figura 4, se puede evidenciar que el $84 \%$ se encuentra en nivel medio, y el $12 \%$ en el nivel alto en el pre test; después se distingue que el $100 \%$ se ubica en nivel alto en el post test. De tal manera, se determina que la propuesta basada en la teoría Kaizen fortalece la competencia interactiva en docentes de Historia y Geografía de Guayaquil.

\section{Objetivo específico 4}

- Verificar que una propuesta basada en la teoría Kaizen, fortalece la competencia investigativa en los docentes de Historia y Geografía en Guayaquil - 2021.

\section{Tabla 5}

Niveles de la dimensión investigativa

Dimensión: Competencia investigativa

\begin{tabular}{ccccc}
\hline \multirow{2}{*}{ NIVEL } & \multicolumn{2}{c}{ PRE TEST } & \multicolumn{2}{c}{ POS TEST } \\
& F & $\%$ & F & $\%$ \\
\hline Alto & 9 & 18 & 50 & 100 \\
Medio & 40 & 80 & 0 & 0 \\
Bajo & 1 & 2 & 0 & 0 \\
Total & 50 & 100 & 50 & 100 \\
\hline
\end{tabular}

Fuente: Cuestionario aplicado

\section{Figura 5}

Niveles de la dimensión investigativa 


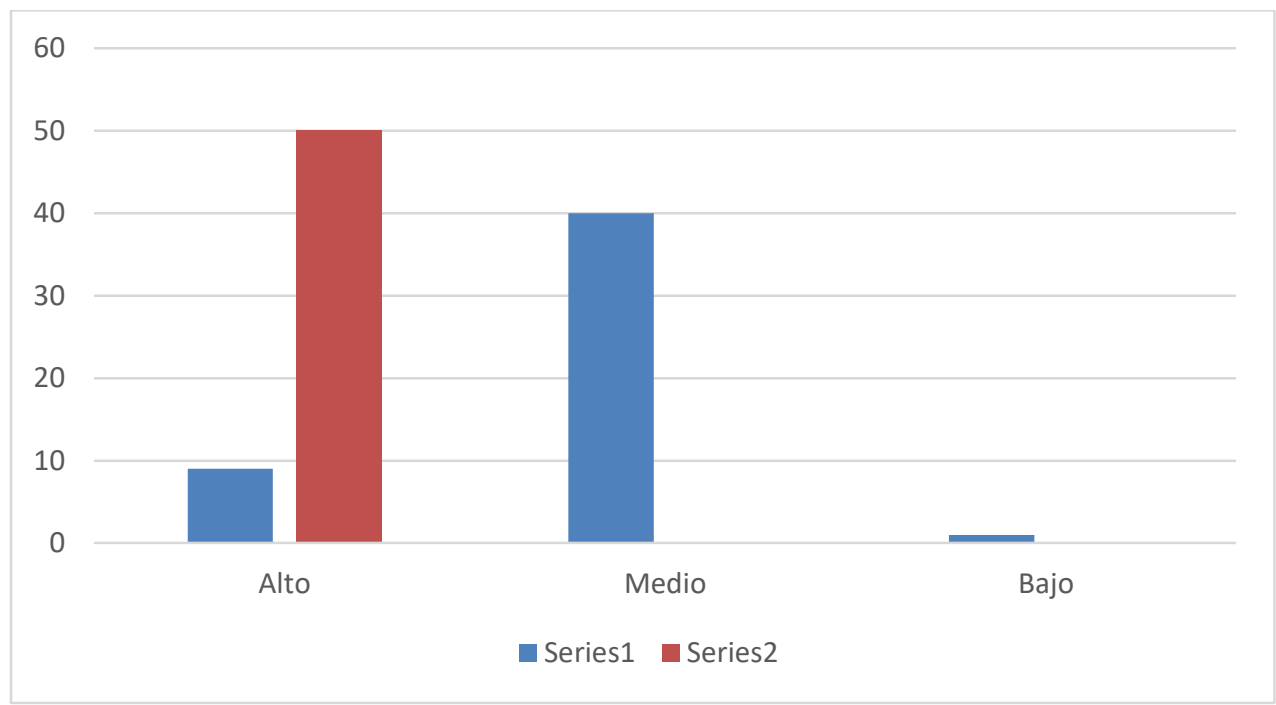

Fuente: Tabla 5

Interpretación: es posible determinar en la tabla 5 y figura 5 como el $80 \%$ se encuentra en nivel medio, y el $18 \%$ en el nivel alto en el pretest; posteriormente se observa que el $100 \%$ se ubica en nivel alto en la post prueba. En base a lo anterior, se concluye que la propuesta basada en la teoría Kaizen fortalece la competencia investigativa en docentes de Historia y Geografía de Guayaquil.

\section{Objetivo específico}

- Determinar que una propuesta basada en la teoría Kaizen, fortalece la competencia para la dirección en los docentes de Historia y Geografía en Guayaquil - 2021.

\section{Tabla 6}

Niveles de la dimensión competencia para la dirección

\begin{tabular}{ccccc}
\hline NIVEL & \multicolumn{2}{c}{ PRE TEST } & \multicolumn{2}{c}{ POS TEST } \\
& F & $\%$ & F & $\%$ \\
\hline Alto & 10 & 20 & 50 & 100 \\
Medio & 40 & 80 & 0 & 0 \\
Bajo & 0 & 0 & 0 & 0 \\
Total & 50 & 100 & 50 & 100 \\
\hline
\end{tabular}

Fuente: Cuestionario aplicado

\section{Figura 6}

Niveles de la dimensión competencia para la dirección 


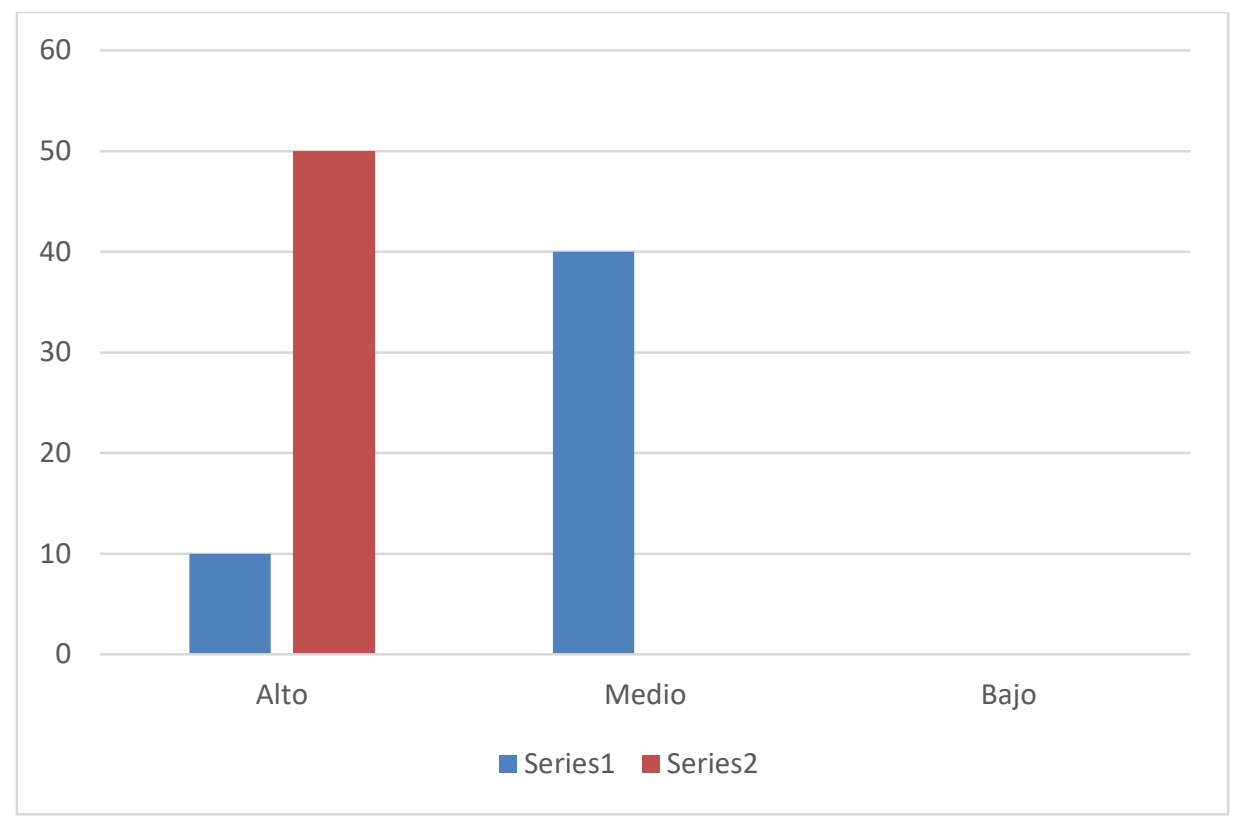

Fuente: Tabla 6

Interpretación: En la tabla 6 y figura 6, se puede ver que el $80 \%$ se encuentra en nivel medio, y el $20 \%$ en el nivel alto en el pre test; luego se denota que el $100 \%$ se ubica en nivel alto en el post test. Por ello, se llega a la conclusión que la propuesta basada en la teoría Kaizen fortalece la competencia para la dirección en docentes de Historia y Geografía de Guayaquil.

\section{Tabla 7}

Prueba de normalidad

\begin{tabular}{lccccccc}
\hline & \multicolumn{3}{c}{ Kolmogorov-Smirnov $^{\text {a }}$} & \multicolumn{3}{c}{ Shapiro-Wilk } \\
& Estadístico & gl & Sig. & Estadístico & gl & Sig. \\
\hline Competencias pedagógicas después &, 111 & 50 &, 172 &, 952 & 50 &, 040 \\
Competencias pedagógicas antes &, 220 & 50 &, 000 &, 919 & 50 &, 002 \\
\hline
\end{tabular}

a. Corrección de significación de Lilliefors

Interpretación: Los datos no provienen de una distribución normal, siendo el nivel de significancia menor a 0,05, para la investigación pre experimental corresponde Wilcoxón.

\section{Análisis inferencial}

Hi: Existe influencia de la propuesta basada en la teoría Kaizen, en el fortalecimiento de las competencias pedagógicas en los docentes de Historia y Geografía en Guayaquil- 
2021.

Ho: No existe influencia de la propuesta basada en la teoría Kaizen, en el fortalecimiento de las competencias pedagógicas en los docentes de Historia y Geografía en Guayaquil 2021.

\section{Tabla 8}

Comparación de medias de las competencias pedagógicas

\section{Estadísticas de muestra única}

\begin{tabular}{lcccc}
\hline & N & Media & $\begin{array}{c}\text { Desviación } \\
\text { estándar }\end{array}$ & $\begin{array}{c}\text { Media de error } \\
\text { estándar }\end{array}$ \\
\hline Competencias pedagógicas después & 50 & 114,96 & 5,631 &, 796 \\
Competencias pedagógicas antes & 50 & 84,46 & 4,432 &, 627 \\
\hline
\end{tabular}

\section{Tabla 9}

Estadística de prueba de la variable competencias pedagógicas

\section{Estadísticos de prueba ${ }^{a}$}

\begin{tabular}{lc}
\hline & $\begin{array}{c}\text { Competencia pedagógicas antes } \\
\text { Competencias pedagógicas después }\end{array}$ \\
\hline $\mathrm{Z}$ & $-6,157^{\mathrm{b}}$ \\
Sig. asintótica (bilateral) &, 000 \\
\hline
\end{tabular}

Interpretación: En la tabla 8 se visualiza diferencia de medias de 30,50, y en la tabla 9, se evidencia que el nivel de significancia es $0,000<0,05$, por tanto, se acepta la hipótesis de investigación (Hi) y se rechaza la hipótesis nula (Ho)

Hi: Existe influencia de la propuesta basada en la teoría Kaizen, en el fortalecimiento de la competencia didáctica en los docentes de Historia y Geografía en Guayaquil - 2021.

Ho: No existe influencia de la propuesta basada en la teoría Kaizen, en el fortalecimiento de la competencia didáctica en los docentes de Historia y Geografía en Guayaquil - 2021.

\section{Tabla 10}

Comparación de medias de la competencia didáctica

\section{Estadísticas de muestra única}




\begin{tabular}{lcccc}
\hline & N & Media & $\begin{array}{c}\text { Desviación } \\
\text { estándar }\end{array}$ & $\begin{array}{c}\text { Media de error } \\
\text { estándar }\end{array}$ \\
\hline Competencia didáctica después & 50 & 21,98 & 1,186 &, 168 \\
Competencia didáctica antes & 50 & 17,38 & 2,710 &, 383 \\
\hline
\end{tabular}

\section{Tabla 11}

Estadística de prueba de la competencia didáctica

\section{Estadísticos de prueba ${ }^{a}$}

\begin{tabular}{lc}
\hline Competencia Didáctica antes \\
Competencia Didáctica después
\end{tabular}

a. Prueba de rangos con signo de Wilcoxon

b. Se basa en rangos positivos.

Interpretación: En la tabla 10 se determina una diferencia de medias de 4,60, también en la tabla 11, se distingue que el nivel de significancia es $0,000<0,05$, por tanto, se acepta la hipótesis de investigación (Hi) y se rechaza la hipótesis nula (Ho)

Hi: Existe influencia de la propuesta basada en la teoría Kaizen, en el fortalecimiento de la competencia productiva en los docentes de Historia y Geografía en Guayaquil - 2021. Ho: No existe influencia de la propuesta basada en la teoría Kaizen, en el fortalecimiento de la competencia productiva en los docentes de Historia y Geografía en Guayaquil 2021.

\section{Tabla 12}

Comparación de medias de la competencia productiva

\section{Estadísticas de muestra única}

\begin{tabular}{lcccc}
\hline & N & Media & $\begin{array}{c}\text { Desviación } \\
\text { estándar }\end{array}$ & $\begin{array}{c}\text { Media de error } \\
\text { estándar }\end{array}$ \\
\hline Competencia productiva después & 50 & 19,10 & 1,147 &, 162 \\
Competencia productiva antes & 50 & 13,18 & 1,480 &, 209 \\
\hline
\end{tabular}

\section{Tabla 13}

Estadística de prueba de la competencia productiva

\section{Estadísticos de prueba ${ }^{a}$}




\begin{tabular}{lc}
\hline $\mathrm{Z}$ & $\begin{array}{c}\text { Competencia Productiva antes } \\
\text { Competencia Productiva después }\end{array}$ \\
\hline Sig. asintótica (bilateral) & $-6,121^{\mathrm{b}}$ \\
&, 000 \\
\hline
\end{tabular}

a. Prueba de rangos con signo de Wilcoxon

b. Se basa en rangos positivos.

Interpretación: En la tabla 12 se evidencia diferencia de medias de 6,08, de la misma manera en la tabla 13 , se constata que el nivel de significancia es $0,000<0,05$, por tal motivo, se acepta la hipótesis de investigación (Hi) y se rechaza la hipótesis nula (Ho)

Hi: Existe influencia de la propuesta basada en la teoría Kaizen, en el fortalecimiento de la competencia interactiva en los docentes de Historia y Geografía en Guayaquil - 2021. Ho: No existe influencia de la propuesta basada en la teoría Kaizen, en el fortalecimiento de la competencia interactiva en los docentes de Historia y Geografía en Guayaquil - 2021

\section{Tabla 14}

Comparación de medias de la competencia interactiva

\section{Estadísticas de muestra única}

\begin{tabular}{lcccc}
\hline & N & Media & $\begin{array}{c}\text { Desviación } \\
\text { estándar }\end{array}$ & $\begin{array}{c}\text { Media de error } \\
\text { estándar }\end{array}$ \\
\hline Competencia interactiva después & 50 & 18,74 & 1,209 &, 171 \\
Competencia interactiva antes & 50 & 13,64 & 1,638 &, 232 \\
\hline
\end{tabular}

\section{Tabla 15}

Estadística de prueba de la competencia interactiva

\section{Estadísticos de prueba ${ }^{a}$}

\begin{tabular}{lc}
\hline & $\begin{array}{c}\text { Competencia Interactiva antes } \\
\text { Competencia Interactiva después }\end{array}$ \\
\hline $\mathrm{Z}$ & $-6,114^{\mathrm{b}}$ \\
Sig. asintótica (bilateral) &, 000 \\
\hline
\end{tabular}

a. Prueba de rangos con signo de Wilcoxon

b. Se basa en rangos positivos.

Interpretación: En la tabla 14 se observa diferencia de medias de 5,50, asimismo en la tabla 15 , se puede apreciar que el nivel de significancia es $0,000<0,05$, así, se acepta la hipótesis de investigación (Hi) y se rechaza la hipótesis nula (Ho)

Hi: Existe influencia de la propuesta basada en la teoría Kaizen, en el fortalecimiento de 
la competencia investigativa en los docentes de Historia y Geografía en Guayaquil - 2021. Ho: No existe influencia de la propuesta basada en la teoría Kaizen, en el fortalecimiento de la competencia investigativa en los docentes de Historia y Geografía en Guayaquil 2021

\section{Tabla 16}

Comparación de medias de la competencia investigativa

\section{Estadísticas de muestra única}

\begin{tabular}{lcccc}
\hline & N & Media & $\begin{array}{c}\text { Desviación } \\
\text { estándar }\end{array}$ & $\begin{array}{c}\text { Media de error } \\
\text { estándar }\end{array}$ \\
\hline Competencia investigativa después & 50 & 25,44 & 2,022 &, 286 \\
Competencia investigativa antes & 50 & 18,66 & 1,533 &, 217 \\
\hline
\end{tabular}

\section{Tabla 17}

Estadística de prueba de las competencia investigativa

\section{Estadísticos de prueba ${ }^{a}$}

\begin{tabular}{lc}
\hline & $\begin{array}{c}\text { Competencia Investigativa antes } \\
\text { Competencia Investigativa después }\end{array}$ \\
\hline $\mathrm{Z}$ & $-6,108^{\mathrm{b}}$ \\
Sig. asintótica (bilateral) &, 000
\end{tabular}
a. Prueba de rangos con signo de Wilcoxon
b. Se basa en rangos positivos.

Interpretación: En la tabla 16 se denota diferencia de medias de 6,78 y en la tabla 17, se aprecia que el nivel de significancia es $0,000<0,05$, por esta razón, se acepta la hipótesis de investigación (Hi) y se rechaza la hipótesis nula (Ho)

Hi: Existe influencia de la propuesta basada en la teoría Kaizen, en el fortalecimiento de la competencia para la dirección en los docentes de Historia y Geografía en Guayaquil 2021.

Ho: No existe influencia de la propuesta basada en la teoría Kaizen, en el fortalecimiento de la competencia para la dirección en los docentes de Historia y Geografía en Guayaquil $-2021$

\section{Tabla 18}

Comparación de medias de la competencia para la dirección 
Estadísticas de muestra única

\begin{tabular}{lcccc}
\hline & N & Media & $\begin{array}{c}\text { Desviación } \\
\text { estándar }\end{array}$ & $\begin{array}{c}\text { Media de error } \\
\text { estándar }\end{array}$ \\
\hline Competencia para la dirección después & 50 & 29,70 & 2,043 &, 289 \\
Competencia para la dirección antes & 50 & 21,60 & 1,979 &, 280 \\
\hline
\end{tabular}

\section{Tabla 19}

Estadística de prueba de la competencia para la dirección

Estadísticos de prueba ${ }^{a}$

\begin{tabular}{lc}
\hline & $\begin{array}{c}\text { Competencia para la dirección antes } \\
\text { Competencia para la dirección después }\end{array}$ \\
\hline $\mathrm{Z}$ & $-6,104^{\mathrm{b}}$ \\
$\mathrm{Sig}$. asintótica (bilateral) &, 000
\end{tabular}

a. Prueba de rangos con signo de Wilcoxon

b. Se basa en rangos positivos.

Interpretación: La tabla 18 permite visualizar una diferencia de medias de 8,10, igualmente en la tabla 19, se evidencia que el nivel de significancia es $0,000<0,05$, por tanto, se acepta la hipótesis de investigación (Hi) y se rechaza la hipótesis nula (Ho)

En relación al objetivo general: Determinar que una propuesta basada en la teoría Kaizen fortalece las competencias pedagógicas en los docentes de Historia y Geografía en Guayaquil - 2021, la tabla 1, evidencia que del $86 \%$ que se encontraba en nivel medio en el pre test, el 100\% pasó a un nivel alto en el post test. Del mismo modo, la tabla 8 deja ver una diferencia del 30,50 entre el pre y postest, también se observa significancia de $0,000<0,05$, por tanto, se acepta la hipótesis de investigación (Hi) y se rechaza la hipótesis nula (Ho).

Tales resultados, concuerdan con Restrepo (2016), donde se concluyó la importancia de potenciar las competencias docentes; manifestando que la universidad tiene el perfil requerido bajo las políticas y requerimientos de la propia institución. La teoría Kaizen es siempre el mejoramiento continuo de las capacidades, tanto conocimientos, habilidades y destrezas. Tal como lo afirma el autor Cheverría, que detalló que "la competencia que posee el docente debería disponer de los conocimientos, destrezas y actitudes necesarias para ejercer la propia actividad laboral, resolver las dificultades de forma independiente, creativa y capacitarse para colaborar en su ambiente de trabajo" (citado por Cruz, 2015: 
página). De la misma forma, se coincide con Escobar (2005), que expresó que las competencias pedagógicas se constituyen en el pilar de la destreza de los educadores, lo cual resulta en la productividad de los saberes de los alumnos. Por su lado, Zapata (2018) de Perú, menciona que existe efectividad al aplicarse propuestas basadas en la metodología Kaizen para fortalecer las competencias en los docentes.

En lo que respecta al objetivo específico 1: Determinar que una propuesta basada en la teoría Kaizen, fortalece la competencia didáctica en los docentes de Historia y Geografía en Guayaquil - 2021. Se distingue en la tabla 2 que el 52\% estaba en nivel medio y el $44 \%$ en nivel alto, del pretest; luego se ubicaron en nivel alto con el $100 \%$ para el 'postest. También, la tabla 10 presenta diferencia de 4,60, a favor del postest, igualmente la tabla 11 , denota nivel de significancia de $0,000<0,05$, por ello, se acepta la hipótesis de investigación (Hi) y se rechaza la hipótesis nula (Ho). En este orden, existe similitud con Pazmiño (2017) que indicó que la capacitación en competencias docentes maximiza los conocimientos en estrategias de enseñanza; igualmente es importante entender el valor de la capacitación docente en competencias pedagógicas para incrementar la calidad en el proceso de enseñanza-aprendizaje. Cabe destacar, que Freire señala que la educación es la acción social que promueve la libertad del ser humano.

En lo que concierne al objetivo específico 2: Comprobar que una propuesta basada en la teoría Kaizen, fortalece las competencias productivas en los docentes de Historia y Geografía en Guayaquil - 2021, del 80\% pasaron al 100\%, del nivel medio a nivel alto, siendo los más altos porcentajes para el postest (Tabla 5), además, en la tabla 12 se observa diferencia del de 6,08, con relación al pre y post test, asimismo presenta significancia de 0,000 < 0,05. Según Gutiérrez (2003), las escuelas se encuentran buscando incesantemente el mejoramiento continuo, por lo que se afirma que el valor se lo da a una comunidad el nivel de capacitación de sus ciudadanos; por esto, debe emplearse una metodología que aperture cambios constantes y palpables que contribuyan a que se cambie la condición del estudiante durante el proceso de enseñanza.

Refiriéndose al objetivo específico 3: Demostrar que una propuesta basada en la teoría Kaizen, fortalece la competencia interactiva en los docentes de Historia y Geografía en Guayaquil - 2021, el 100\% se ubicó en nivel alto del postest después de haber estado en $84 \%$ de nivel medio en el pre test. Además, existe diferencia de 5,50, entre pre y post test (Tabla 15). También, en la tabla 15 , se observa que el nivel de significancia es $0,000<$ 
0,05, por tanto, se acepta la hipótesis de investigación (Hi) y se rechaza la hipótesis nula (Ho). Se encuentran similitudes con la investigación de Miyashiro (2017) puesto que se deben implementar planes de mejora continua para aumentar el desempeño profesional, tomando en cuenta la interactuación entre los miembros de la organización. Por su parte, Guerrero (2018) concluye que los procesos de mejoramiento continuo, benefician el aseguramiento de la calidad educativa al interior de las organizaciones; igualmente, contribuyen como instrumento para impulsar el pensamiento crítico y el aprendizaje de los educandos.

Relacionado con el objetivo específico 4: Verificar que una propuesta basada en la teoría Kaizen, fortalece la competencia investigativa en los docentes de Historia y Geografía en Guayaquil - 2021, la tabla 5 muestra que el $80 \%$ estaba en nivel medio, posteriormente se ubicaron nivel alto con $100 \%$, del pre al postest. También se evidencia diferencia del 6,78 (Tabla 16) y nivel de significancia de $0,000<0,05$. Esto quiere decir que la propuesta basada en Teoría Kaizen si influye en la mejora de la competencia para la dirección.

Finalmente, el aplicar los conceptos de la teoría mencionada, impacta de manera positiva y significativa en cada dimensión de la variable, en palabras de Arellano (2017), es verdadero ese cambio ya que se emplea $5 \mathrm{~S}$, las cuales se desarrollan de manera consecutiva (SEIRI (sentido de utilización) - SEITON (sentido de organización) - SEISO (sentido de limpieza) - SEIKETSU (sentido de normalización); lo anterior se relaciona con las competencias pedagógicas que fueron clasificadas por Castellanos (2005) y Braslavsky (1999) citados por Aguiar y Rodríguez (2018).

Finalmente, en cuanto al objetivo específico 5: Determinar que una propuesta basada en la teoría Kaizen, fortalece la competencia para la dirección en los docentes de Historia y Geografía en Guayaquil - 2021. La tabla 6, presenta que el 80\% estaba en nivel medio (pretest) paso después al 100\% (postest). A su vez, se presenta en la tabla 18 la diferencia del 8,10, entre el pre y pos test, como también el nivel de significancia es $0,000<0,05$. Se puede demostrar que la gestión de las instituciones escolares con base en las funciones de un director, así como la de un educador, no pueden limitarse a velar por el cumplimiento de los planes, programas, aprendizajes y facilidades que brindan las instituciones de educación superior; como resultado, deberán ocuparse de crear una organización donde sus procesos de trabajo sean eficientes y efectivos, por ello el presente trabajo de investigación emplea la aplicación de nuevas prácticas de enseñanza basadas 
en la metodología Kaizen, que han brindado estrategias educativas novedosas utilizadas en la interacción docente-aprendizaje, y que han servido como canal del aprendizaje de los estudiantes.

\section{CONCLUSIÓN O CONSIDERACIONES FINALES}

1) La propuesta influye positivamente en el fortalecimiento de competencias pedagógicas, evidenciado en la tabla 1 , y tabla 8 , donde se observa que los valores del postest fueron superiores al pre test, aceptándose la hipótesis de investigación (Hi) y rechazándose la hipótesis nula (Ho).

2) La propuesta fortalece la competencia didáctica en docentes, con evidencia en la tabla 10, presentando valores mayores para el postest que los del pre test. Quedando demostrado que se acepta la hipótesis de investigación (Hi) y se rechaza la hipótesis nula (Ho)

3) La propuesta, fortalece la competencia productiva en docentes demostrado en la tabla 3 , y además la tabla 12 muestra diferencia sustantiva entre el pre y postest, por lo que se acepta la hipótesis de investigación (Hi) y se rechaza la hipótesis nula (Ho).

4) La propuesta, tiene significancia ya que fortalece la competencia interactiva en docentes, evidenciando que el 100\% se ubicó en nivel alto del postest. De la misma manera existe influencia por lo que se afirma que se acepta la hipótesis de investigación (Hi) y se rechaza la hipótesis nula (Ho)

5) La propuesta, fortalece la competencia investigativa en docentes (Tabla 5) al considerar que el $100 \%$ se ubicó en nivel alto del postest, igualmente se evidencia diferencia del 6,78 (Tabla 16). Se acepta la hipótesis de investigación (Hi) y se rechaza la hipótesis nula (Ho).

6) La propuesta, fortalece la competencia para la dirección en los docentes (Tabla 6) del $80 \%$ del nivel medio paso después al $100 \%$ del nivel alto. A su vez presenta diferencia del 8,10; demostrando que la propuesta influye significativamente; se acepta la hipótesis de investigación (Hi) y se rechaza la hipótesis nula (Ho).-

\section{Estas conclusiones, permiten deducir las siguientes recomendaciones:}

1) Aplicar la propuesta basada en teoría Kaizen a los docentes de otras materias de estudio, con la finalidad de fortalecer el desarrollo de las competencias pedagógicas.

2) Aplicar la propuesta basada en la teoría Kaizen en los docentes de instituciones privadas, para conocer la diferencia con las instituciones públicas. 
3) Ampliar la muestra para una segunda investigación de la variable, permitiendo contextualizar el constructo en estudio.

4) Aplicar la propuesta basada en la teoría Kaizen en los docentes de educación primaria, para conocer el nivel de competencias pedagógicas que poseen.

5) Aplicar la propuesta basada en la teoría Kaizen, en los educadores cuyos estudiantes tengan habilidades diferentes, para conocer el nivel de competencias pedagógicas que ellos tienen y cómo pueden mejorar su metodología de enseñanza.

\section{REFERENCIAS BIBLIOGRÁFICAS}

Aguiar Santiago, X.; Rodríguez Pérez, L. (2018). La formación de competencias pedagógicas en los profesores universitarios. EDUMECENTRO 2018; 10(2):141-159 ISSN 2077-2874 RNPS 2234. Universidad Central "Marta Abreu” de Las Villas: Villa Clara-Cuba. Recuperado de: https://bit.ly/3GYkguQ

Alarcón, J. (2017). Modelo de mejora continua basado en procesos y su impacto en la calidad de los servicios que perciben los clientes de la empresa de servicios ServiFreno de la ciudad de Quito - Ecuador. Universidad Nacional Mayor de San Marcos [Tesis de Doctorado]. Recuperado de: https://bit.ly/327sMcl

Álvarez, A. (2014). Biografías narrativas de docentes: experiencias de adversidad educativa, significados y capacidades. Caso Colegio Freinet de Guayaquil. Guayaquil: Casa Grande.

Arellano, E. (2017). Metodología de las 5S. Unión Social de Empresarios de México: Ciudad Juárez-México. Recuperado de https://docplayer.es/33247599Metodologia-de-las-5s-u-s-e-m-edgar-arellano.html

Arias, F. (2005). El Proyecto de Investigación: Introducción a la Metodología Científica. Caracas, Venezuela: Editorial Episteme, c. a.

Arias, E. (10 de diciembre de 2020). Investigación Aplicada. Economipedia.com [Mensaje en un blog]. Recuperado de: https://economipedia.com/definiciones/investigacion-aplicada.html

Bizneo blog (2021). ¿Qué es la metodología 5S? [Mensaje en un blog]. Recuperado de https://www.bizneo.com/blog/que-es-la-metodologia-5s/

Bruns, B. \& Luque, J. (2014). Docentes excelentes: Cómo mejorar el aprendizaje en América Latina y el Caribe, resumen. Washington, DC, Banco Mundial. 
Licencia: Creative Commons de Reconocimiento CC BY 3.0. Publicado originalmente en inglés. Disponible en: https://bit.ly/3sDt1Xt

Escobar, M. (2005). Las Competencias Laborales: ¿La estrategia laboral para la competitividad de las organizaciones? Cali, Colombia. Recuperado de: https://bit.ly/3qe6sWh

Escobar, I. M. C., \& Adames, C. P. (2008). Competencias docentes de profesores de pregrado: diseño y validación de un instrumento de evaluación. Universitas Psychologica, 7(2), 456-456.

Escobar-Pérez, J. y Cuervo-Martínez, A. (2008). Validez de contenido y juicio de expertos: una aproximación a su utilización. En Avances en Medición, 6, pp. 2736. Recuperado de: https://bit.ly/3Flu0yQ

Espinoza, E. \& Campuzano, J. (2019). Training by competences of teachers of basic and high education. Conrado, 15(67), 250-258. Epub 02 de junio de 2019. Recuperado en 15 de octubre de 2021, de https://bit.ly/33MuAI6

Freire, P. (1998). Pedagogía de la autonomía. Saberes necesarios para la práctica educativa. México: Editorial Siglo XXI.

Freire, P. (2006). Pedagogía de la autonomía. España: Siglo veintiuno editores.

Gamero, A. (23 de octubre de 2019). 10 razones por las que estudiar Geografía e Historia. La Piedra de Sísifo. Gabinete de Curiosidades. [Mensaje en un blog]. Recuperado de: https://bit.ly/3Eh4ut2

Guerrero, E. (2018). El Kaizen como proceso de mejora continua, en el aseguramiento de la calidad de las instituciones educativas superiores del Ecuador, periodo 2015-2016. Universidad Nacional Mayor de San Marcos [Tesis de Doctorado]. Recuperado de https://cybertesis.unmsm.edu.pe/handle/20.500.12672/7555

Guerrero, F. (2021). Dirección en un Colegio de Pedagogía Williams. En prensa.

Gómez, L. (2005). Comparación de una propuesta pedagógica universitaria con las prácticas cotidianas en las aulas. Revista Mexicana de Investigación Educativa. 10 (24), 165 -189. Recuperado de: https://bit.ly/3Ei50XM

Gómez, M. (26 de febrero de 2020). Teoría Kaizen. [Mensaje en un blog]. Recuperado de http://mabegolu-a.blogspot.com/2008/05/teoria-kaisen.html

Gutiérrez, O. (2003). El Proceso Educativo desde los enfoques centrados en el Aprendizaje. Trabajo de Investigación. Recuperado de: https://bit.ly/3H2xEOq 
Hernández, R., Fernández, C., \& Baptista, P. (2003). Metodología de la Investigación (3era Ed.). México D.F.: Mcgraw-HILL.

Hernández, R., Fernández, C., \& Baptista, P. (2010). Metodología de la Investigación. México D.F.: Mcgraw-HILL / Interamericana Editores, S.A. de C.V.

Imai, M. (1986). Kaizen: La clave para el éxito competitivo de Japón. New York: McGraw Hill.

Imai, M. (1989). Kaizen, la clave de la ventaja competitiva japonesa. México, D.F.: CECSA.

Imai, M. (1997). Gemba Kaizen. New York, NY: Mcgraw-Hill.

Imai, M. (1998). Cómo implementar el Kaizen en el sitio de trabajo. (Edición en español). Editorial: McGraw-Hill Spanish.

Instituto Nacional de Evaluación Educativa (2018). La educación en Ecuador: logros alcanzados y nuevos desafíos. Resultados educativos 2017-2018 (primera ed.). Quito, Ecuador. Recuperado de: https://bit.ly/3yMqnPR

Kaizen Institute. Masaaki Imai Biografía, el padre de la filosofía Kaizen [en línea]. Recuperado de:

<http://es.kaizen.com/fileadmin/DATA/kaizen_es/Biograf\%C3\%ADa\%20Masaaki\%20 Imai.pdf>

Martínez, J. (2008). La ciudadanía se convierte en competencia: Avances y retrocesos. En J. Gimeno Sacristán (Coord.), Educar por competencias, ¿Qué hay de nuevo? (pp.103-142). Madrid: Morata. Disponible en: https://bit.ly/3qhQPNF

Matamoros, C. (2016). El desarrollo de las habilidades macrointelectuales en el contexto de un currículo abierto: Caso Colegio Freinet de Guayaquil. Guayaquil: As.

Méndez, C. (2001). Metodología, diseño y desarrollo del proceso de investigación (3era Ed.). Bogotá: Mcgraw-HILL.

Ministerio de Educación Nacional (2013). Competencias TIC para el desarrollo profesional docente. Imprenta Nacional: Corporación Colombia Digital.

Miyashiro, E. (2017). Aplicación de la metodología Kaizen o mejora continua y el desempeño organizacional del instituto de educación superior tecnológico privado CEPEA. Universidad Inca Garcilaso de la Vega-Perú. [Tesis de Maestría]. Recuperado de: http://repositorio.uigv.edu.pe/handle/20.500.11818/4071 
Moreno, M. (2011). El Método Kaizen para el Mejoramiento Continuo. [Mensaje en un blog]. Recuperado de https://www.elblogsalmon.com/conceptos-deeconomia/el-metodo-kaizen-para-el-mejoramiento-continuo

Morles, V. (2004). Planeamiento y Análisis de Investigación. Caracas: El Dorado.

Muñoz, D. (2017). La educación como práctica de la libertad: una lectura antropológico-pedagógica al pensamiento de Paulo Freire. Dialnet, Vol. 9, N 1, pp 26-41, ISSN: 2027-2391. Recuperado de: https://bit.ly/3mo2hWH

Pazmiño, J. (2017). Capacitación sobre la competencia pedagógica y el conocimiento de estrategias de enseñanza de los docentes de la Universidad Laica Vicente Rocafuerte de Guayaquil - Ecuador, 2016. Universidad Nacional Mayor de San Marcos [Tesis de Doctorado]. Recuperado de: https://bit.ly/3H2e05q

Reglamento General a la Ley Orgánica de Educación Superior expedido mediante Decreto Ejecutivo Nro. 865, de 01 de septiembre de 2011, publicado en el Registro Oficial Suplemento Nro. 526, Quito, 02 de septiembre de 2011.

Rengifo Ávila, G. K., \& Espinoza Freire, E. E. (2019). Estudio sobre los problemas en la educación ecuatoriana y políticas educativas, 1990-2018. Revista Metropolitana de Ciencias Aplicadas, 2(3), 175-182. Recuperado de: http://remca.umet.edu.ec/index.php/REMCA

Restrepo, J. (2016). Las competencias del docente de posgrados: una perspectiva desde la formación de programas de maestría. Universidad Autónoma de BarcelonaColombia [Tesis de Doctorado]. Recuperado de: https://bit.ly/3J7IbKc

Ricoy, C. (2006). Contribución sobre los paradigmas de investigación. Revista do Centro de Educação, 31 (1), 11-22.

Rivadeneira Rodríguez, E. (2017). Competencias didácticas-pedagógicas del docente, en la transformación del estudiante universitario. Orbis. Revista Científica Ciencias Humanas, 13(37),41-55. [fecha de Consulta 24 de mayo de 2021]. ISSN: 18561594. Disponible en: https://www.redalyc.org/articulo.oa?id=70952383003

Rodríguez, I. \& De la Vega, R. (2018). Revisión nacional e internacional de las competencias profesionales de los docentes de Educación Física. Retos, $\mathrm{N}^{\circ} 34$, ISSN: Edición impresa: 1579-1726. Edición Web: 1988-2041. Federación Española de Asociaciones de Docentes de Educación Física. Recuperado de: https://bit.ly/3H5iybd 
Rojas C. \& Salazar S. (2019). Aplicación de la metodología 5's para la optimización en la gestión del almacén en una empresa importadora de equipos de laboratorio. Recuperado de: https://bit.ly/3J66ok0

Sampieri-Hernández, R., Fernández-Callado, C., \& Baptista-Lucio, P. (2010). Metodología de la Investigación. México: McGraw-Hill.

Serrano, J. (2013). Administración y diseño del curriculum. Monografias.com. Recuperado de: https://bit.ly/3GZ53JY

Suasnabas, L. y Juárez, J. (2020). Calidad de la educación en Ecuador. ¿Mito o realidad? Vol. 6, núm. 2, abril-junio 2020, pp. 133-157. Dom. Cien., ISSN: 2477-8818. Recuperado de: https://bit.ly/3qdJcI7

Tamayo y Tamayo, M. (2006). Técnicas de Investigación. (2 ${ }^{\mathrm{a}}$ Edición). México: Editorial Mc Graw Hill.

UNESCO (2021). Docentes. [Mensaje en un blog]. Recuperado de: https://es.unesco.org/themes/docentes

Vasco, C. (2011). Pedagogía, formación, currículo y didáctica. Colección de la Pedagogía Colombiana/Iberoamericana. Bogotá, Colombia: Redipe

Williams, L. (2010). Ir, llegar y volver: Lectura en acción. Proyecto participante del Cuarto Concurso de Excelencia Educativa 2010 organizado por la Fundación para la Investigación y Desarrollo para América Latina. Quito: FIDAL, 2010.

Williams, L. (2018). Percepciones sobre la Incidencia de un Bachillerato con Currículo Abierto en el Desempeño Universitario: Caso Colegio Freinet de Guayaquil. En prensa.

Yépez (2015). La rendición de cuentas pedagógicas ante los padres y miembros de la comunidad en el Colegio Freinet de Guayaquil. Guayaquil: As.

Zapata, G. (2018). Modelo De Gestión Organizacional basado en la Teoría del Kaizen para mejorar el Desempeño Docente en la Institución Educativa $N^{\circ} 10828$ Chiclayo. Universidad César Vallejo, Perú. [Tesis de Doctorado]. Recuperado de: https://repositorio.ucv.edu.pe/handle/20.500.12692/34521

Bernstein, A. (1964). A Handbook of Statistics Solutions for the Behavioral Sciences. New York: Holt, Rinehart \& Winston.

Messick, S. (1998). Test validity: A matter of consequence. Social Indicators Research 45 (1-3): 35-44. 
Nunnally, J. C. (1967). Psychometric theory. New York: Mc Graw - Hill.

Stevens, S. (1951). Handbook of Experimental Psychology. New York: J. Wiley and Sons Inc.

UNESCO (2008). ICT Competency Standards for Teachers. Recuperado de: http://unesdoc.unesco.org/images/0015/001562/156207e.pdf 